\title{
Enfermedad de Osgood-Schlatter: deporte, adolescencia y dolor
}

\author{
I. Carabaño Aguadoa, L. Llorente Otones ${ }^{b}$

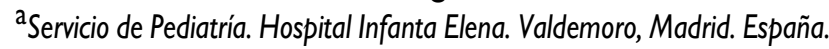 \\ ${ }^{b}$ Departamento de Pediatría. Hospital de Fuenlabrada. Fuenlabrada, Madrid. España.
}

\begin{abstract}
Resumen
La enfermedad de Osgood-Schlatter es una de las causas más frecuentes de gonalgia en niños mayores y adolescentes deportistas. Es la consecuencia de la tracción ejercida por el tendón rotuliano sobre el centro de osificación de la tuberosidad tibial anterior. Los cambios radiológicos incluyen condensación, fragmentación, aparición de osículos y edema de partes blandas. Su tratamiento puede ser iniciado por parte de los pediatras de Atención Primaria, e incluye analgesia, estiramientos, potenciación del cuádriceps y modificación de la práctica deportiva.
\end{abstract}

Palabras clave: Enfermedad de Osgood-Schlatter. Apofisitis tibial. Osteocondrosis.

Osgood-Schlatter disease: sports, adolescence and pain

\section{Abstract}

Osgood-Schlatter's disease is one of the most common causes of knee pain in older children and adolescent athletes. It is the result of the traction by the patellar tendon on the centre of ossification of the anterior tibial tuberosity. Radiographic changes include condensation, fragmentation, appearance of ossicles and soft tissue edema. The treatment may be initiated by primary care pediatricians, including analgesia, stretching, quadriceps strengthening and modification of the sport.

Key words: Osgood-Schlatter disease. Apophysitis tibialis. Osteochondrosis.

\section{Caso clínico}

Paciente de 13 años de edad que acude al Servicio de Urgencias Pediátricas de nuestro hospital, aquejado de dolor en ambas rodillas de un año de evolu- ción (más acusado en el lado izquierdo), con empeoramiento progresivo hasta hacerse prácticamente invalidante en la última semana. El dolor es de características mecánicas, y empeora claramente

Iván Carabaño Aguado, carabano1975@hotmail.com

Los autores declaran no presentar conflictos de intereses en relación con la preparación y publicación de este artículo. 
con la actividad física, especialmente cuando juega al baloncesto. La exploración física muestra una tumoración dolorosa sobre ambas tuberosidades tibiales anteriores (TTA), sin calor ni rubor asociados. Sin datos de inestabilidad o derrame articular. Se solicitó estudio radiológico (figuras 1 y 2), en cuya proyección lateral se objetivó una discontinuidad de la tuberosidad tibial anterior, junto con heterogeneidad en la densidad de la misma y fragmentación ósea. Todo ello es compatible con la enfermedad de Osgood-Schlatter (EOS).

\section{Discusión}

A pesar de que el deporte practicado de forma regular y con moderación es un hecho reconocido como beneficioso para la salud física y social, no siempre deporte y salud son sinónimos. Los principales factores desfavorables que se deben tener en cuenta en este sentido son: presencia del cartílago de crecimiento, debilidad muscular y el estrés excesivo que a veces supone la competición en sí1,2. Si nos centramos en el plano físico y en los traumatismos debidos a una solicitación excesiva del sistema

Figura 1. Enfermedad de Osgood-Schlatter. Densidad radiológica irregular y despegamiento de la cortical en ambas tibias.

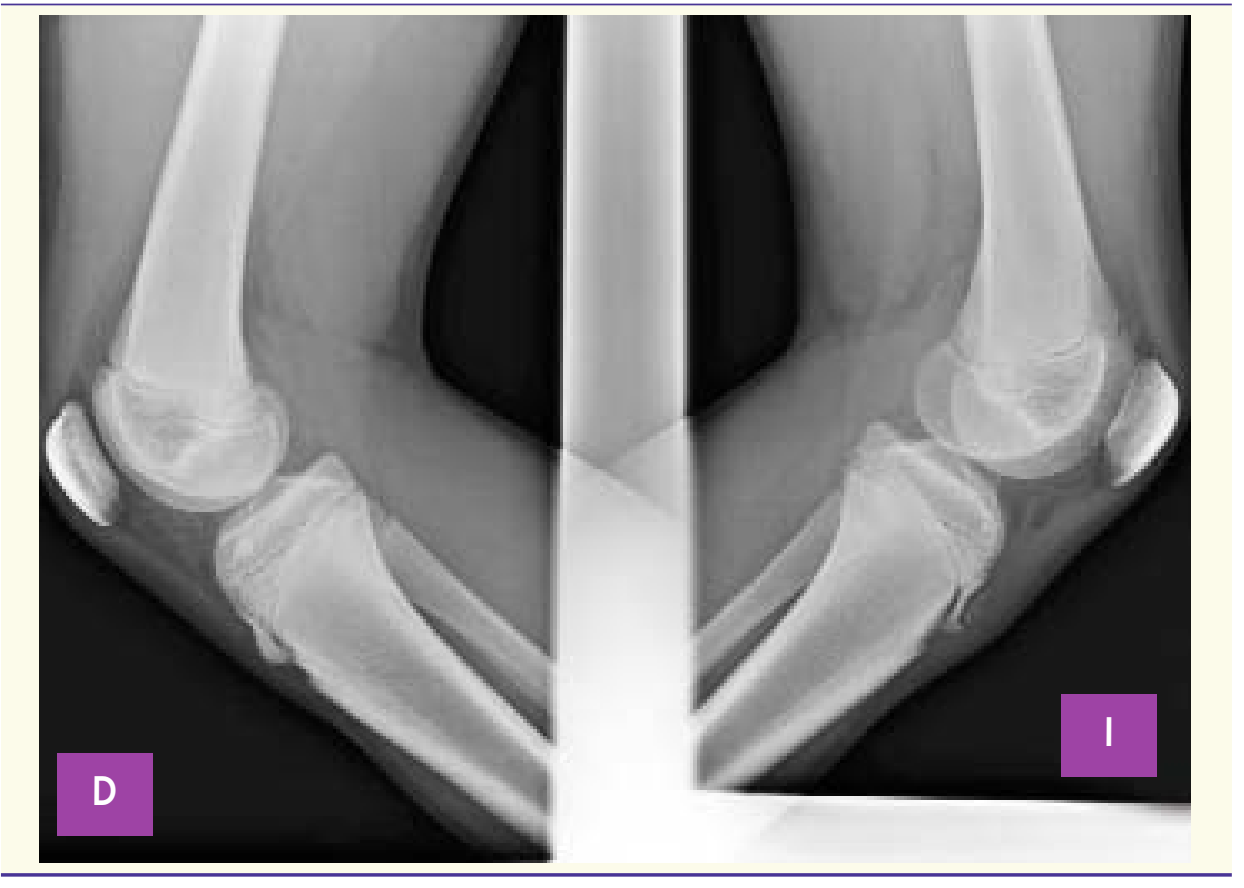


Figura 2. Enfermedad de Osgood-Schlatter. Detalle de la fragmentación de la apófisis tibial derecha.

musculoesquelético, a lo largo de la infancia y adolescencia son frecuentes la EOS, el dolor femororrotuliano, la apofisitis del calcáneo, la espondilolisis y la osteoatritis disecante, así como el codo y el hombro de "la pequeña liga"2,3.

La EOS, descrita hace más de 100 años, es una de las causas principales de gonalgia en adolescentes de 10-15 años de edad. Un estudio reciente asegura que afecta al 9,8\% de la población en este rango etario. Es más frecuente en el sexo masculino, en los pacientes con sobrepeso y en los que tienen un recto femoral corto. Se asocia con deportes que impliquen saltos múl- tiples, carreras cortas y patadas, como baloncesto, fútbol, voleibol, salto de altura, salto de longitud y gimnasia deportiva. En cuanto a su patogenia, cabe incluir a la EOS dentro del grupo de las apofisitis por tracción. La repetitiva secuencia de contracción-relajación del cuádriceps genera de forma incesante una tracción de la tuberosidad tibial anterior por parte del tendón rotuliano. La avulsión crónica del hueso producida por este tendón genera un auténtico callo de fractura y el ensanchamiento de la tuberosidad. Esta puede llegar a fracturarse, con lo cual el dolor se exacerba llamativamente ${ }^{1-4}$. 


\begin{tabular}{l|l}
\hline Tabla 1. Hallazgos exploratorios en la enfermedad de Osgood-Schlatter \\
\hline Maniobras que despiertan dolor & $\bullet$ Presión sobre la TTA \\
& $\bullet$ Extensión de la rodilla \\
& $\bullet$ Contrarresistencia \\
& $\bullet$ Flexión pasiva forzada \\
\hline Palpación característica & $\bullet$ Tumefacción nodular en la TTA \\
\hline TTA: tuberosidad tibial anterior.
\end{tabular}

Desde el punto de vista de la exploración física, podemos objetivar los puntos que se detallan en la tabla $1^{1-4}$.

El diagnóstico de esta entidad es esencialmente clínico, si bien el examen radiológico permite descartar otros orígenes del dolor. Los hallazgos se exponen en la tabla $2^{3,5}$.

Dado que la EOS suele tener una evolución favorable, el tratamiento inicial es conservador, y se puede divulgar desde las consultas pediátricas de Atención Primaria. Este incluye administración de analgésicos/antiinflamatorios no esteroideos, aplicación de frío local, descarga de la articulación a través de una pérdida controlada de peso si el paciente es obeso y reposo relativo (exclusión de la actividad física "violenta"). Dado que esta medida suele ser mal percibida por niños mayores y adolescentes, se recomienda una modificación parcial de la práctica deportiva que desempeñan. Por ejemplo, se les debe invitar a que practiquen natación y ciclismo en llano sin restricciones. Por otro lado, antes de practicar deportes que impliquen carrera o saltos, se les aconseja hacer a diario estiramientos del aparato extensor de la pierna, así como potenciación isométrica del cuádriceps ${ }^{6,7}$. Tan solo un $10 \%$ de los pacientes tiene una mala evolución

\begin{tabular}{|c|c|}
\hline Partes blandas & $\begin{array}{l}\text { - Edema generalizado } \\
\text { - Borrado de la grasa infrarrotuliana } \\
\text { - Engrosamiento del tendón rotuliano }\end{array}$ \\
\hline Hueso & $\begin{array}{l}\text { - Condensación } \\
\text { - Fragmentación } \\
\text { - Reestructuración } \\
\text { - Osificación tibial irregular } \\
\text { - Aparición de osículos independientes } \\
\text { - Ensanchamiento óseo } \\
\text { - Imagen en gota de cera de la epífisis tibial superior }\end{array}$ \\
\hline
\end{tabular}


que precise liberación quirúrgica y exéresis de los restos osiculares o cartilaginosos libres ${ }^{8,9}$. En los últimos años, esta se realiza con éxito a través de artrosco$\mathrm{pia}^{10}$. En una serie publicada recientemente, después de la cirugía un $87 \%$ de los pacientes pudo llevar una vida nor- mal y un $75 \%$ era capaz de realizar ejercicio físico al mismo nivel que el de partida9.

La principal complicación de la EOS a largo plazo se conoce como enfermedad de Pinlach-Aznar, y cursa con dolor crónico por fallo en la osificación de la TTA ${ }^{11}$.

\section{Bibliografía}

1. Cassas KJ, Cassettari-Wayhs A. Chilhood and adolescent sports-related overuse injuries. Am Fam Physician. 2006;73:1014-22.

2. Stein CJ, Micheli LJ. Overuse injuries in youth sports. Phys Sportsmed. 2010;38:102-8.

3. Davis KW. Imaging pediatric sports injuries: lower extremity. Radiol Clin North Am. 2010;48: 1213-35.

4. De Lucena $G L$, Gomes CD, Guerra RO. Prevalence and associated factors of Osgood-Schlatter syndrome in a population-based simple of Brazilian adolescents. Am J Sports Med. 2010;12. Epub ahead of print.

5. Gottsegen CJ, Eyer BA, White EA, Learch TJ, Forrester D. Avulsion fractures of the kene: imaging findings and clinical significance. Radiograph. 2008;28:1755-70.

6. Gholve PA, Scher DM, Khakharia S, Widmann RF, Green DW. Osgood-Schlatter syndrome. Curr Opin Pediatr. 2007;19:44-50.
7. Bloom OJ, Mackler L, Barbee J. Clinical inquiries. What is the best treatment for Osgood-Schlatter disease? J Fam Pract. 2004;53:153-6.

8. Pihlajamäki HK, Visuri TI. Long-term outcome after surgical treatment of unresolved OsgoddSchaltter disease in young men: surgical technique. J Bone Joint Surg Am. 2010;92:258-64.

9. Pihlajamäki HK, Mattila VM, Parviainen $M$, Kiuru MJ, Visuri TI. Long-term outcome after surgical treatment of unresolved Osgodd-Schaltter disease in young men. J Bone Joint Surg Am. 2009; 91:2350-8.

10. Beyzadeouglu T, Inan M, Bekler H, Altintas F. Artrhoscopic escisión of an ununited ossicle due to Osgood-Schlatter disease. Arthroscopy. 2008; 24:1081-3.

11. Orgaz-Gallego MP, Tricio-Armero MJ. Enfermedad de Osgood-Schlatter: a propósito de dos casos. SEMERGEN. 2009;35:418-20. 